\title{
Reversible bone pain and symmetric bone scan uptake in a dialysis patient treated with cinacalcet: a case report
}

\author{
Oliver Lenz 1 , Rhea Sancassani2 , Carla Bottino ${ }^{3}$ and Alessia Fornoni*1,4
}

\begin{abstract}
Introduction: The medical management of secondary hyperparathyroidism in patients with end-stage renal disease involves a combination of dietary restrictions, phosphate binders, active vitamin D analogs, and calcimimetics.

Case presentation: We report the case of a 36-year-old Hispanic dialysis patient, originally from Cuba and now residing in the USA, who developed severe bone pain and muscle twitching after starting low dose cinacalcet, despite normal pre-dialysis ionized calcium and elevated parathyroid hormone. The clinical symptoms correlated with increased symmetrical uptake on bone scan that resolved rapidly upon discontinuation of cinacalcet.
\end{abstract}

Conclusion: Cinacalcet may induce severe bone pain and a unique bone scan uptake pattern in hemodialysis patients.

\section{Introduction}

The medical management of secondary hyperparathyroidism (SHPT) in patients with end-stage renal disease (ESRD) involves a combination of dietary restrictions, phosphate binders, active vitamin D analogs, and calcimimetics. Treatment is aimed at achieving the goals delineated in the Kidney Disease Outcomes Quality Initiative in order to avoid the adverse mineral metabolic abnormalities, including renal bone disease and cardiovascular mortality.

Cinacalcet (Sensipar, Amgen, USA), a type II calcimimetic, allosterically increases the sensitivity of the calcium-sensing receptor, lowering the threshold for activation, and thereby decreasing secretion of parathyroid hormone (PTH) [1]. Studies have demonstrated its ability to lower PTH without significantly increasing serum calcium, phosphorus, or the $\mathrm{Ca} \times \mathrm{P}$ product [2]. Although symptomatic hypocalcemia may be a concern in pre-dialysis patients treated with cinacalcet [3], we only found one case report of cinacalcet-induced symptomatic hypocalcemia in a dialysis patient. This was accompanied by bone pain and thought to be a result of hungry bone syndrome caused by the too rapid correc-

\footnotetext{
* Correspondence: afornoni@med.miami.edu

${ }^{1}$ Division of Nephrology and Hypertension, University of Miami Miller School of Medicine, Miami, Florida, USA

Full list of author information is available at the end of the article
}

tion of PTH [4], similar to what can be observed in a patient with severe SHPT following parathyroidectomy or kidney transplantation. In contrast, we report the case of a patient who developed severe bone pain and muscle twitching after starting low dose cinacalcet, despite normal pre-dialysis ionized calcium and elevated PTH.

\section{Case presentation}

The patient is a 36-year-old Hispanic man, originally from Cuba and now residing in the USA, with hypertension and ESRD secondary to focal segmental glomerulosclerosis who had been undergoing hemodialysis for the past two years using a right radiocephalic fistula. He presented to a community hospital complaining of excruciating, constant, bilateral pain in both legs below the knees and the left forearm. He described the pain as dull and aching with periods of sharp non-radiating pain that required treatment with high dose narcotics. A ${ }^{99 \mathrm{~m}} \mathrm{Tc}$ bone scan performed on initial presentation showed bilateral symmetric uptake in the proximal tibia and distal femur, increased uptake in the metacarpophalangeal joints of the left hand, and bilateral radial and ulnar uptake. The axial skeleton and ribs were not involved. Although these findings were consistent with a metabolic rather than an inflammatory or infectious process and all blood cultures were negative, he received intravenous vancomycin and gentamycin for presumed osteomyelitis 
because he had undergone laparascopic cholecystectomy for acute cholecystitits and gram-negative sepsis four weeks prior to this admission. After 12 days of treatment our patient's pain had progressed in intensity and he transferred his care to our facility.

Our patient's past medical history was significant for focal segmental glomerulosclerosis leading to ESRD, hypertension, a seizure disorder, anemia of chronic disease, and SHPT. His medications included nifedipine, metoprolol, levetiracetam, cetirizine, calcium acetate, and cinacalcet; the latter had been started four weeks prior to the admission when our patient's intact PTH concentration in the serum was $750 \mathrm{pg} / \mathrm{ml}$. The patient was now taking $30 \mathrm{mg}$ daily.

At the time he presented to our institution our patient's pain continued. It was associated with fasciculations of the face and extremities, intermittent fevers, generalized weakness, and diarrhea. Physical examination showed a blood pressure of 170/95, heart rate of 105 beats per minute, respiratory rate of 18 per minute, temperature of $38.4^{\circ} \mathrm{C}$, and $99 \%$ oxygen saturation on room air. Otherwise, his examination was only remarkable for muscle fasciculations in the face and upper extremities and a functioning right radiocephalic fistula.

Laboratory data revealed a white blood cell count of 5.8 $\times 10^{3} / \mu \mathrm{L}\left(6 \times 10^{9} / \mathrm{L}\right)$ with normal differential, hemoglobin $11.6 \mathrm{~g} / \mathrm{dL}(116 \mathrm{~g} / \mathrm{L})$, and platelets $267 \times 10^{3} / \mu \mathrm{L}(267 \times$ $\left.10^{9} / \mathrm{L}\right)$. Serum chemistries were significant for blood urea nitrogen $(B U N) 60 \mathrm{mg} / \mathrm{dL}(21.4 \mathrm{mmol} / \mathrm{L})$, creatinine 11.1 $\mathrm{mg} / \mathrm{dL}(846 \mu \mathrm{mol} / \mathrm{L})$, calcium $8.7 \mathrm{mg} / \mathrm{dL}(2.17 \mathrm{mmol} / \mathrm{L})$, ionized calcium $1.06 \mathrm{mEq} / \mathrm{L}(0.53 \mathrm{mmol} / \mathrm{L})$, phosphorus $6.8 \mathrm{mg} / \mathrm{dL}(2.2 \mathrm{mmol} / \mathrm{L})$, albumin $3.3 \mathrm{~g} / \mathrm{dL}(33 \mathrm{~g} / \mathrm{L})$, alkaline phosphatase 465IU/L, PTH 659 pg/ml (659 ng/L). A Doppler ultrasound of the upper extremity did not reveal a deep venous thrombosis. X-rays of our patient's legs and left arm did not reveal any pathology.

Pain control was attempted with intravenous morphine but remained suboptimal. Given his intermittent fevers, blood cultures and stool studies were requested, and a bone scan was ordered to evaluate for osteomyelitis. All blood cultures remained negative, but stool studies were positive for Clostridium difficile toxin. Based on the clinical picture, a presumptive diagnosis of pseudomembranous colitis due to use of broad-spectrum antibiotics was made, and he was subsequently treated with oral metronidazole leading to resolution of his fevers. The $99 \mathrm{~m} \mathrm{Tc}$ bone-scan again showed an abnormal pattern of uptake involving symmetric bilateral upper and lower extremities with no involvement of the axial skeleton (Figure 1A). With the intent to perform a bone biopsy a computed tomography scan of our patient's lower extremities was performed, which did not reveal any focal findings. Similarly, magnetic resonance imaging of his lower extremi-

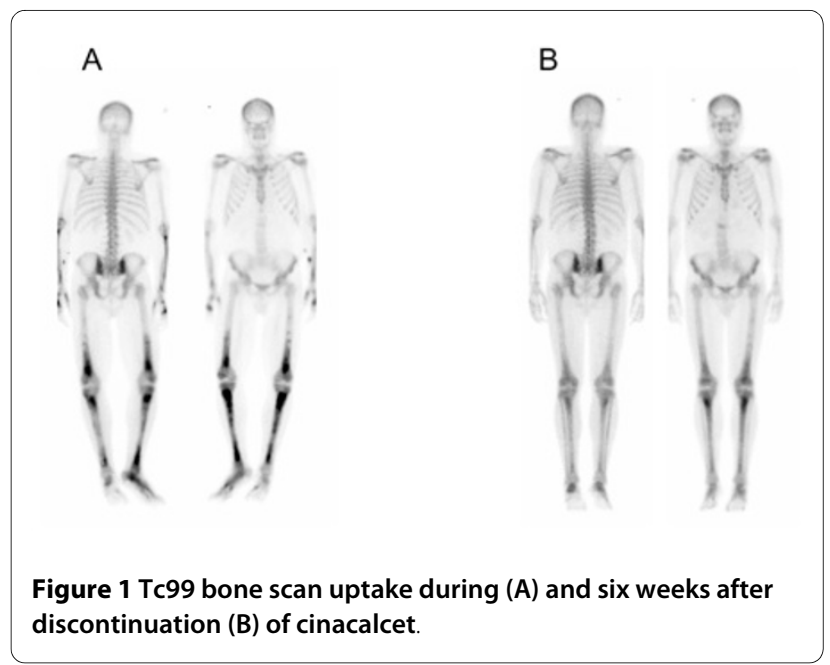

ties showed a preserved bony cortex with no focal findings.

Several metabolic conditions were considered to explain the ${ }^{99 \mathrm{~m} T c}$ uptake pattern [5]. However, the clinical presentation of our patient was inconsistent with any of the most plausible diagnoses, such as Paget's disease, rheumatoid arthritis, hypervitaminosis $\mathrm{D}$, or osteomalacia. Our patient underwent hemodialysis three times weekly using a bicarbonate-based dialysate with a calcium concentration of $1.25 \mathrm{mmol} / \mathrm{L}$. He achieved an adequate clearance, as measured by a $\mathrm{Kt} / \mathrm{V}$ of 1.2 or greater, making uremic neuropathy an unlikely explanation, and his neurological examination did not suggest a neuropathic picture, which is why nerve conduction studies were not performed. Similarly, renal osteodystrophy appeared unlikely given the very symmetrical uptake. Given that calcium-sensing receptors have been reported to be present in bone, we hypothesized that cinacalcet may have caused increased osteoblastic activity, and consequently cinacalcet was stopped on day eight of hospitalization. Two days after discontinuation our patient's pain resolved and he was weaned off all analgesia. Muscle fasciculation also resolved in the absence of any change in ionized calcium, which had remained normal. Vitamin D analogues were prescribed for the management of his secondary hyperparathyroidism at discharge. A repeat bone scan six weeks later showed none of the abnormalities seen on the previous studies (Figure 1B). His PTH had decreased and the serum calcium stayed normal. At his six-week follow-up visit after discharge, he remained pain free.

\section{Conclusions}

This case demonstrates that adverse effects from treatment with a calcium-receptor agonist should be considered in the differential diagnosis when evaluating a hemodialysis patient presenting with severe bone pain. 
Calcium-sensing receptors (CaSR) can sense the extracellular calcium ion concentration and enable key tissues to maintain calcium homeostasis. Their expression in the proximal tubule, distal convoluted tubule, thick ascending limb of Henle, and chief cells of the parathyroid gland has been described and fully characterized. However, more recent data suggest novel localization of CaSRs on human arteries, breast cancer cells, as well as bones [6]. While it has been thought that a change in extracellular calcium concentration is necessary to activate CaSRs, the localization of CaSR on the membrane of the endoplasmic reticulum and the Golgi apparatus [7] suggests that CaSR may be involved in the regulation of intracellular calcium distribution independently of the extracellular calcium. A recent animal model with a constitutively active CaSR in osteoblasts suggests that CaSR activity may be independent of systemic changes in serum calcium or PTH concentrations [8]. Thus, it may be possible that our patient developed muscle fasciculations as a direct consequence of intracellular calcium redistribution despite a normal serum ionized calcium concentration. Similarly, the findings of symmetrical increase in osteoblastic activity by bone scan in the presence of normal ionized calcium may be explained by the presence of agonistic activity of cinacalcet on bone CaSR irrespective of the calcium concentration. In fact, it has been recently shown that CaSR may play a pivotal role in the control of both osteoclast and osteoblast differentiation as well as in the localization of hematopoietic progenitor stem cells to the bone endosteal surface. The evidence of cinacalcet induced increase in bone mineral density in long bones but not in the lumbar spine is consistent with the findings we have observed in our patient by bone scan [9]. It is possible that a specific pattern of CaSR expression in our patient has been responsible for the described findings. These could not be verified by polymerase chain reaction (PCR) on a bone biopsy specimen since our patient refused the procedure while hospitalized and there was no reason to perform a biopsy at a later point once he was completely asymptomatic. Our patient did not receive any active forms of vitamin D while taking cinacalcet. This is noteworthy given that almost all patients on maintenance hemodialysis receive active vitamin D sterols, and that hemodialysis patients who participated in past clinical trials with cinacalcet received concomitant treatment with vitamin D. It is conceivable that the lack of vitamin D therapy contributed to our patient's presentation. Unfortunately, we were unable to follow the patient long-term and hence we do not know if the patient was re-challenged with cinacalcet after starting an active vitamin D analog.

This case does not prove definitive evidence for a causal relationship between our patient's bone pain, abnormal bone scan, and cinacalcet use. Nevertheless, we believe that the clinician faced with a similar constellation should at least consider the possibility that cinacalcet may induce severe bone pain and a unique bone scan uptake pattern in hemodialysis patients.

\section{Consent}

Written informed consent could not be obtained despite all reasonable attempts. Every effort has been made to protect the identity of the patient and there is no reason to think that the patient or their family would object to this publication.

\section{Editor's note}

This manuscript was submitted prior to our change of policy on consent.

Competing interests

The authors declare that they have no competing interests.

\section{Authors' contributions}

$\mathrm{RS}$ and $\mathrm{CB}$ compiled and analyzed the clinical data. RS, CB, OL, and AF were major contributors in writing the manuscript. All authors read and approved the final manuscript.

\section{Acknowledgements}

We thank Dr Mark Gelbard from the Department of Internal Medicine for critical discussions and Dr Serafini Aldo from the Department of Radiology for providing the images.

\section{Author Details}

1Division of Nephrology and Hypertension, University of Miami Miller School of Medicine, Miami, Florida, USA, ${ }^{2}$ Department of Medicine, Jackson Memorial Hospital, Miami FL, USA, 3Universidad San Martin de Porres, Lima, Peru and ${ }^{4}$ Diabetes Research Institute, University of Miami Miller School of Medicine, Miami, Florida, USA

Received: 21 October 2009 Accepted: 24 June 2010

Published: 24 June 2010

\section{References}

1. Valle C, Rodriguez M, Santamaria R, Almaden Y, Rodriguez ME, Canadillas S, Martin-Malo A, Aljama P: Cinacalcet reduces the set point of the PTH calcium curve. J Am Soc Nephrol 2008, 19:2430-2436.

2. Fishbane S, Shapiro WB, Corry DB, Vicks SL, Roppolo M, Rappaport K, Ling $X$, Goodman WG, Turner S, Charytan C: Cinacalcet $\mathrm{HCl}$ and concurrent low-dose vitamin D improves treatment of secondary hyperparathyroidism in dialysis patients compared with vitamin $D$ alone: the ACHIEVE study results. Clin J Am Soc Nephrol 2008, 3:1718-1725.

3. Chonchol M, Locatelli F, Abboud HE, Charytan C, de Francisco AL, Jolly S, Kaplan M, Roger SD, Sarkar S, Albizem MB, Mix TC, Kubo Y, Block GA: A randomized, double-blind, placebo-controlled study to assess the efficacy and safety of cinacalcet $\mathrm{HCl}$ in participants with CKD not receiving dialysis. Am J Kidney Dis 2009, 53:197-207.

4. Lazar ES, Stankus N: Cinacalcet-induced hungry bone syndrome. Semin Dial 2007, 20:83-85.

5. Buckley O, O'Keeffe S, Geoghegan T, Lyburn ID, Munk PL, Worsley D, Torreggiani WC: 99 mTc bone scintigraphy superscans: a review. NuCl Med Commun 2007, 28:521-527.

6. Yamaguchi T: The calcium-sensing receptor in bone. J Bone Miner Metab 2008, 26:301-311.

7. Tu CL, Chang W, Bikle DD: The role of the calcium sensing receptor in regulating intracellular calcium handling in human epidermal keratinocytes. J Invest Dermato/ 2007, 127:1074-1083.

8. Dvorak MM, Chen TH, Orwoll B, Garvey C, Chang W, Bikle DD, Shoback DM: Constitutive activity of the osteoblast $\mathrm{Ca} 2+-$-sensing receptor promotes loss of cancellous bone. Endocrinology 2007, 148:3156-3163. 
9. Lien $Y H$, Silva AL, Whittman D: Effects of cinacalcet on bone mineral density in patients with secondary hyperparathyroidism. Nephrol Dial Transplant 2005, 20:1232-1237.

doi: $10.1186 / 1752-1947-4-191$

Cite this article as: Lenz et al., Reversible bone pain and symmetric bone

scan uptake in a dialysis patient treated with cinacalcet: a case report Journal of Medical Case Reports 2010, 4:191

Submit your next manuscript to BioMed Central and take full advantage of:

- Convenient online submission

- Thorough peer review

- No space constraints or color figure charges

- Immediate publication on acceptance

- Inclusion in PubMed, CAS, Scopus and Google Scholar

- Research which is freely available for redistribution

Submit your manuscript at www.biomedcentral.com/submit 